\title{
Fine needle aspiration cytology-based accurate and rapid diagnosis of breast tuberculosis masquerading as a case of abscess
}

\author{
Vityala Yethindra ${ }^{1}$, Altynai Zhumabekova ${ }^{2}$, Cholpon Dzhumakova ${ }^{3}$, Tugolbai Tagaev ${ }^{4}$, \\ Asel Namazbekova ${ }^{3}$, and Bolotbek Djanaliev ${ }^{4}$ \\ ${ }^{1}$ International Higher School of Medicine \\ ${ }^{2}$ City Maternity Hospital No. 2 \\ ${ }^{3}$ National Center of Oncology and Hematology \\ ${ }^{4}$ I K Akhunbaev Kyrgyz State Medical Academy
}

February 13, 2021

\begin{abstract}
We are reporting a case of breast tuberculosis (BT) that was initially misdiagnosed as a breast abscess. Fine needle aspiration cytology (FNAC) helped in diagnosing BT faster by avoiding different invasive diagnostic methods. FNAC can be used as the primary diagnostic method of choice for the diagnosis of BT.

Fine needle aspiration cytology -based accurate and rapid diagnosis of breast tuberculosis masquerading as a case of abscess

Vityala Yethindra ${ }^{*}$, Altynai Zhumabekova ${ }^{2}$, Cholpon Dzhumakova ${ }^{3}$, Tugolbai Tagaev ${ }^{4}$, Asel Namazbekova ${ }^{5}$, Bolotbek Djanaliev 6

${ }^{1}$ Department of Pathology, International Higher School of Medicine, International University of Kyrgyzstan, Bishkek, Kyrgyzstan

${ }^{2}$ Obstetrician-gynecologist, City Maternity Hospital No. 2, Bishkek, Kyrgyzstan

${ }^{3}$ Department of Gastroenterology, National Center of Oncology and Hematology, Bishkek, Kyrgyzstan

${ }^{4}$ Department of Public Health and Healthcare, I.K. Akhunbaev Kyrgyz State Medical Academy, Bishkek, Kyrgyzstan

${ }^{5}$ Department of Cancer registry, National Center of Oncology and Hematology, Bishkek, Kyrgyzstan

${ }^{6}$ Department of Pathological Anatomy, I.K. Akhunbaev Kyrgyz State Medical Academy, Bishkek, Kyrgyzs$\tan$
\end{abstract}

Running title: Fine needle aspiration cytology as the primary diagnostic method of breast tuberculosis

*Corresponding author: Vityala Yethindra, Department of Pathology, International Higher School of Medicine, International University of Kyrgyzstan, Bishkek, Kyrgyzstan, Email:yethindravityala10@gmail.com, Contact: +91 9121925658

\section{ABSTRACT}

We are reporting a case of breast tuberculosis (BT) that was initially misdiagnosed as a breast abscess. Fine needle aspiration cytology (FNAC) helped in diagnosing BT faster by avoiding different invasive diagnostic methods. FNAC can be used as the primary diagnostic method of choice for the diagnosis of BT. 
Keywords: Breast tuberculosis, Fine needle aspiration cytology, Antitubercular therapy.

\section{Key clinical message}

In patients with breast tuberculosis (BT), fine-needle aspiration cytology functions as a primary diagnostic method to guide the appropriate management of initial earlier in the course and helps in preventing the wider spread of BT.

\section{INTRODUCTION}

In 1829, the earliest breast tuberculosis (BT) case was reported by Sir Astley Cooper and mentioned as "scrofulous swelling of the bosom". ${ }^{1} \mathrm{BT}$ is a rare and uncommon presentation of tuberculosis ${ }^{2}$ with an $0.1 \%$ overall incidence in breast lesions in developed and $4 \%$ in surgically treated lesions in developing countries. ${ }^{3,4}$ $\mathrm{BT}$ is excluded and at times over-diagnosed as breast cancer or abscess. ${ }^{5,6} \mathrm{BT}$ is recognized substantially in women of reproductive age, lactating, multiparous, and sometimes also seen in men $(4.5 \% \text { of cases })^{4,7}$; it coexists with immunodeficiency states like HIV ${ }^{8}$ Primary BT is very rare ${ }^{9}$ and secondary BT arises due to hematogenous, retrograde (axillary lymph nodes), or direct spread (lung, pleura, mediastinum, and articular lesions). ${ }^{10}$ In this study, we describe a case of an immunocompetent patient with no evidence of any other tubercular foci in the body.

The objective of this report was to emphasize the significance of fine-needle aspiration cytology (FNAC) as the primary diagnostic method of choice in the diagnosis of BT and the avoidance of different invasive diagnostic methods.

\section{CASE PRESENTATION}

A 34-year-old woman presented to our hospital, Clinical Hospital of the President Association and the Government of the Kyrgyz Republic, Bishkek, with complaints of a unilateral right painless breast lump of 2 months' duration. The initially painless lump became painful with induration of the skin, ulcer formation, and purulent discharge (pus) from the sinus on the right breast for 20 days before hospitalization. She denied a history of weight loss, night sweats, loss of appetite, and respiratory symptoms. There was no family history of breast cancer, tuberculosis, or other pulmonary diseases. The systemic examination was normal.

Local examination of the right breast revealed a $1.0-\mathrm{cm}$ sinus in the upper outer quadrant with a $2 \times 2-\mathrm{cm}$ palpable lump, and purulent discharge was noted. There were no clinical manifestations or abnormalities in the left breast or nipple-areolar area and no signs of nipple discharge.

Except for the elevated erythrocyte sedimentation rate $(102 \mathrm{~mm} / \mathrm{h})$, her laboratory panel was within normal limits. The patient underwent computed tomography of the neck, abdomen, and breast, which showed a $2 \times 2$ cm lump in the right breast. Unilateral axillary lymphadenopathy was noted on the right side with multiple nodes ranging from 10 to $20 \mathrm{~mm}$ in size. Further evaluation of the right breast with ultrasound examination revealed a hypoechoic lesion with heterogeneous formation $(87 \times 17 \mathrm{~mm})$ with elongated and irregular borders. Doppler ultrasonography (US) revealed elevated circumferential vessels in the central avascular lesions as a sign of existing infection, and a diagnosis of breast abscess was made. ${ }^{9}$ However, there was no symptom alleviation even after a week of co-amoxiclav (1000 mg) every $12 \mathrm{~h}$.

For a definitive diagnosis, the patient underwent FNAC of the right breast lump. Touch imprint smears prepared from the pus revealed acid-fast bacilli (AFB) on Ziehl-Neelsen (ZN) staining. Histological study of tissue pieces revealed the presence of Langhans giant cells, epithelioid cell granulomas, caseous necrosis, lymphocytes, necrotic foci, and fibroblast clusters. Mycobacterium tuberculosis complex was confirmed by a cartridge-based nucleic acid amplification test sensitive to rifampicin in the FNAC sample.

Excision of the fibrous tract and resection of the rib with pus and necrotized tissue were performed. The patient was started on standard antitubercular therapy (ATT) with 2-month regimen (isoniazid, rifampicin, pyrazinamide, and ethambutol), followed by 4-month regimen (isoniazid and rifampicin). She consistently visited the hospital for follow-up for 1 year, and no disease recurrence was noted. 


\section{DISCUSSION}

Historically, clinical manifestations of BT have been observed within 1 year, but they range from months to years. ${ }^{11} \mathrm{BT}$ commonly affects women aged from $20-50$ years, ${ }^{4,12}$ which is equivalent to the higher prevalence of pulmonary tuberculosis seen in a similar age women. ${ }^{13}$ In our report, the patient was a 34-year-old woman presenting with symptoms for 2 months.

BT regularly presents as a lump ${ }^{14}$ in the central or upper outer quadrant of the breast. ${ }^{15}$ In our patient, the lump was present in the upper outer quadrant of the right breast. Manifestation is inconsistent, frequently as a round nodular lump with tissue induration, with fistula formation, but they are infrequently connected with pain. Our patient had diffuse ulceration, pain, and a lump in the right breast.

Tubercular ulcers over the breast skin and tubercular breast abscess with or without discharging sinuses are even common forms of presentation of BT. ${ }^{13}$ Our patient presented with a unilateral draining sinus.

BT was classified earlier as: Nodular, disseminated, sclerosing, obliterans, and acute miliary tubercular mastitis. ${ }^{14}$ The sclerosing, obliterans, and miliary types are of historical significance and currently BT could be reclassified as nodular, disseminated, or abscess. The BT in our patient belonged to the abscess variety.

BT lesions have no specific US findings and are visualized as heterogeneous, hypoechoic, irregularly bordered with internal echoes. ${ }^{16}$ Diagnosis is optimally based on the confirmation of AFB in the breast tissue by ZN staining. ${ }^{17}$ In this study, Doppler US showed features of breast abscess, while FNAC showed epithelioid granulomas with AFB positivity on ZN staining.

FNAC is a trustworthy diagnostic procedure, in which aspirated material is subjected to staining for revealing $\mathrm{AFB}^{18}$ and diagnosing BT. ${ }^{17}$ In BT cases, FNAC evaluation has accurately diagnosed the cases as BT in $73 \%$ of the cases evaluated using histopathology as having both epithelioid granulomas and necrosis. ${ }^{19}$ In this study, histology revealed the presence of Langhans giant cells, epithelioid cell granulomas, and caseous necrosis; FNAC was diagnostically useful for BT.

Trepan biopsy showed a decent positive result. However, inci-/excisional biopsy of breast lumps, ulcers, and sinuses, or a suspected tubercular breast abscess cavity wall mostly diagnoses BT. ${ }^{17,19}$ In this patient, an excision biopsy was suggested, but the patient and her attendants refused the procedure.

Even though tests such as QuantiFERON-TB Gold-In tube have high sensitivity (97.9\%) and specificity $(98.1 \%),{ }^{20}$ there are many limitations such as false-negative results in extrapulmonary tuberculosis $(28.8 \%)^{21}$ and inability to discriminate latent tuberculosis from active tuberculosis infection. ${ }^{22}$ In this study, FNAC evaluation paved the way for an accurate diagnosis.

BT could show a diagnostic difficulty in radiological and microbiological diagnosis, and consequently, high degree of suspicion are required. BT is treatable with $\mathrm{ATT},{ }^{23}$ and surgical intervention is needed in rare situations.

\section{ACKNOWLEDGMENTS}

The authors acknowledge their patient for kind cooperation and for providing consent.

\section{FINANCIAL SUPPORT}

No financial support was received for the study.

\section{CONFLICT OF INTEREST}

The authors declare no conflicts of interest.

\section{AUTHOR CONTRIBUTIONS}

Conception, design of the work, manuscript preparation, and data acquisition: Vityala Yethindra, Tugolbai Tagaev, Asel Namazbekova, Clinical management: Altynai Zhumabekova, Cholpon Dzhumakova, and Manuscript preparation and data acquisition: Vityala Yethindra, Tugolbai Tagaev, Bolotbek Djanaliev. 


\section{REFERENCES}

1. Cooper A. Illustrations of the Diseases of the Breast. London: Longman, Rees, Orme, Brown and Green; 1829.

2. Kalac N, Ozkan B, Bayiz H, Dursun AB, Demirag F. Breast tuberculosis.Breast . 2002; 11(4): 346-349.

3. Akcakaya A, Eryilmaz R, Sahin M, Ozkan OV. Tuberculosis of the breast.Breast J . 2005; 11: 85-86.

4. Tewari M, Shukla HS. Breast tuberculosis: Diagnosis, clinical features and management. Indian J Med Res . 2005; 122: 103-110.

5. Morino GF, Rizzardi G, Gobbi F, Baldan M. Breast tuberculosis mimicking other diseases. Trop Doct . 2007; 37: 177-178.

6. Borens-Fefer B, Engohan-Aloghe C, Noel JC, Simon P, Bucella D, Boutemy R. Primary mammary tuberculosis presenting as voluminous abscess.JBR-BTR . 2008; 91: 54-57.

7. Jaideep C, Kumar M, Khanna AK. Male breast tuberculosis.Postgrad Med J. 1997; 73: 428-429.

8. Hartstein M, Leaf HL. Tuberculosis of the breast as a presenting manifestation of AIDS. Clin Infect Dis. 1992; 15: 692-693.

9. Zandrino F, Monetti F, Gandolfo N. Primary tuberculosis of the breast. A case report. Acta Radiol. 2000; 41: 61-63.

10. Gupta PP, Gupta KB, Yadav RK., Agarwal D. Tuberculous mastitis: A review of seven consecutive cases. Indian J Tuber. 2003; 50: 47.

11. Dubey MM, Agarwal S. Tuberculosis of the breast. J Indian Med Assoc. 1968; 51: 358-359.

12. Hale JA, Peters GN, Cheek JH. Tuberculosis of the breast: Rare but still extant: Review of the literature and report of an additional case. Am J Surg. 1985; 150:620-624.

13. Shukla HS, Kumar S. Benign breast disorders in nonwestern populations: Part II - Benign breast disorders in India. World $J$ Surg. 1989; 13: 746-749.

14. Alagaratnam TT, Ong GB. Tuberculosis of the breast. Br J Surg.1980; 67: 125-126.

15. Bhatt GM, Austin HM. CT demonstration of empyema necessitatis. J Comput Assist Tomogr. 1985; 9:1108-1109.

16. Tuncbilek N, Karakas HM, Okten OO. Imaging of granulomatous mastitis: assessment of three cases. Breast. 2004; 13: 510-514.

17. Kakkar S, Kapila K, Singh MK, Verma K. Tuberculosis of the breast: A cytomorphologic study. Acta Cytol. 2000; 44: 292-296.

18. Harris SH, Khan MA, Khan R, Haque F, Syed A, Ansari MM. Mammary tuberculosis: analysis of thirty-eight patients. ANZ J Surg.2006; 76: 234-237.

19. Shinde SR, Chandawarkar RY, Deshmukh SP. Tuberculosis of the breast masquerading as carcinoma: a study of 100 patients. World J Surg. 1995; 19: 379-381.

20. Takasaki, J., Manabe, T., Morino, E., et al. Sensitivity and specificity of QuantiFERON-TB Gold Plus compared with QuantiFERON-TB Gold In-Tube and T-SPOT.TB on active tuberculosis in Japan. $J$ Infect Chemother. 2018; 24(3): 188-192.

21. Kim YJ, Kang JY, Kim SI, Chang MS, Kim YR, Park YJ. Predictors for false-negative QuantiFERONTB Gold assay results in patients with extrapulmonary tuberculosis. BMC Infect Dis. 2018; 18(1): 457.

22. Ndzi EN, Nkenfou CN, Gwom LC, Fainguem N, Fokam J, Pefura Y. The pros and cons of the QuantiFERON test for the diagnosis of tuberculosis, prediction of disease progression, and treatment monitoring. Int J Mycobacteriol. 2016; 5: 177-184.

23. Luh SP, Hsu JD, Lai YS, Chen SW. Primary tuberculous infection of breast: experiences of surgical resection for aged patients and review of literature. J Zhejiang Univ Sci B. 2007; 8: 580-583. 